Irwin Reiss • Eva Landmann • Matthias Heckmann • Björn Misselwitz $\cdot$ Ludwig Gortner

\title{
Increased risk of bronchopulmonary dysplasia and increased mortality in very preterm infants being small for gestational age
}

Received: 17 September 2002 / Accepted: 21 February 2003 / Published online: 8 April 2003

(C) Springer-Verlag 2003

\begin{abstract}
Objective: The objective was to evaluate the impact of being born small for gestational age (SGA) on neonatal mortality and neonatal pulmonary morbidity in preterm infants $<32$ weeks of gestation. Methods: We reviewed the data reported prospectively to the quality assurance program of the Federal State of Hesse, Germany, from 1990 to 1996 of infants <32 weeks of gestation. SGA was defined as birth weight below the 10th percentile. Mann Whitney $U$ tests were used to compare continuous variables and Fisher's exact tests to analyze differences in dichotomous variables between preterm SGA neonates and preterms born appropriate for gestational age (AGA). The effect of SGA and other potential risk factors for neonatal death and bronchopulmonary dysplasia, i.e., requiring a fraction of inspired oxygen $>0.21$ at day 28 , was tested by multivariable analyses. Results: Data from 1,365 infants were analyzed. One hundred and eighty-three neonates were SGA (mean [SD] birth weight 789 [179] g; mean [SD] gestational age 28.9 [1.7] weeks) and 1,182 were AGA (mean [SD] birth weight 1,260 [348] g; mean [SD] gestational age 28.8 [2.1] weeks). Neonatal mortality and the rate of bronchopulmonary dysplasia were significantly higher in SGA neonates (23 vs. $11 \%$ and 28 vs. $14 \%$, respectively). There was a statistically significant association of SGA with neonatal death (odds ratio $[\mathrm{OR}]=4.54,95 \%$ confidence interval $[\mathrm{CI}] 2.56,8.04)$ and bronchopulmonary dysplasia $(\mathrm{OR}=3.80,95 \%$ CI $2.11,6.84)$. Conclu-
\end{abstract}

Dedicated to Prof. Dr. med. Wolfgang Künzel on occasion of his 65 th birthday

I. Reiss · E. Landmann ( $)$ M. Heckmann · L. Gortner Pediatric Center, Department of Pediatrics and Neonatology, Justus-Liebig-University,

Feulgenstrasse 12, 35385 Giessen, Germany

e-mail: Eva.Landmann@paediat.med.uni-giessen.de

Tel.: +49-641-9943400

Fax: +49-641-9943419

B. Misselwitz

Institute of Quality Assurance,

Hesse, Germany sion: SGA neonates below 32 weeks gestation are a highrisk group regarding neonatal mortality and neonatal pulmonary morbidity.

Keywords Intrauterine growth restriction - Small for gestational age - Respiratory distress syndrome · Bronchopulmonary dysplasia $\cdot$ Prematurity

\section{Introduction}

Neonates born with a birth weight below the 10th percentile generally are classified as small for gestational age (SGA) infants. In most cases this condition is attributed to an intrauterine growth restriction. SGA infants represent a significant percentage of infants admitted to neonatal intensive care units. This especially holds true for SGA infants $<32$ weeks gestational age in whom an increased incidence of neonatal complications was reported recently $[4,8]$. However, studies focusing on the influence of SGA on neonatal morbidity associated with prematurity showed controversial results.

With regard to neonatal pulmonary morbidity, data from the 1970s and 1980s suggested lower rates of respiratory distress syndrome (RDS) in very preterm SGA neonates [13]. In contrast, several recent studies revealed no increased risk of RDS [2, 8, 14] or even a significantly increased risk of RDS [4] in very preterm SGA neonates. Up to now, only two studies focusing on the risk of developing bronchopulmonary dysplasia have been published. Both demonstrate an increased risk of bronchopulmonary dysplasia in very preterm SGA infants compared with preterm neonates being born appropriate for gestational age (AGA) [2, 8]. Recently, Egreteau et al. [6] showed being born SGA to be one of the major risk factors for chronic oxygen dependency in infants born before 32 weeks gestation.

Therefore, our aim was to evaluate neonatal mortality and pulmonary morbidity in very preterm neonates being born SGA compared with AGA preterms obtained from a large representative study sample. 


\section{Materials and methods}

We examined the morbidity and mortality in SGA neonates compared with AGA neonates both being born after $<32$ weeks of gestation using the perinatal and neonatal databases recorded by the quality assurance system of the Federal State of Hesse, Germany, from January 1990 to December 1996. All Hessian hospitals are obliged to report perinatal data to the Hessian quality assurance system center. Obstetrical data, e.g., prenatal corticosteroid administration, preterm premature rupture of membranes (PROM), Apgar score, and umbilical artery $\mathrm{pH}$, have to be reported by the institution where the child was born. If a newborn has to be transferred to a pediatric hospital for further neonatal care, neonatal data have to be reported by the institution the child was transferred to, using an additional questionnaire. Thus for each child two datasets are recorded independently. Trained physicians fill out independently from each other the perinatal, as well as the neonatal datasets. Both, the neonatal and the perinatal datasets have to be sent to the Hessian quality assurance system center. We connected both different datasets. All variables that had to be recorded in the neonatal as well as in the perinatal questionnaire, i.e., date and time of birth, calculated time of birth, gestational age, single or multiple gestation, zip code, gender, and birth weight, had to coincide completely. The sets of data, which did not completely coincide in all these variables, were excluded from the study. Also, we excluded all datasets that were filled out incompletely. We expected a relatively high "drop-out" rate due to incomplete data in either the perinatal or the neonatal dataset. Thus, in a second step, we compared the variables gestational age, being SGA, RDS, bronchopulmonary dysplasia, and mortality between all infants born below 32 weeks of gestation and the infants born below 32 weeks of gestation whose perinatal and neonatal datasets could be connected. Moreover, we compared the variables gestational age, RDS, bronchopulmonary dysplasia, and mortality between all SGA infants below 32 weeks gestation and the SGA infants below 32 weeks gestation whose perinatal and neonatal datasets could be connected.

Infants with lethal malformations and chromosomal aberrations were excluded from the study. Multiple gestations were not excluded from the study, but the variables "twin" and "multiple pregnancy $\geq 3$ " were included in the multivariable analyses identifying risk factors for being born SGA, for neonatal mortality, and for bronchopulmonary dysplasia.

Perinatal variables were defined as follows: The calculation of gestational age was based on maternal menstrual history or early prenatal ultrasound. SGA was defined as birth weight below the 10th percentile according to the percentiles published by Voigt et al. [17] for German newborns. Preterm PROM was diagnosed when the membranes had ruptured more than $12 \mathrm{~h}$ before birth. Preeclampsia was diagnosed according to the criteria established by the American College of Obstetricians and Gynecologists [1]. A complete course of prenatal corticosteroids (two doses of betamethasone) was assumed if the first dose was administered $\geq 24 \mathrm{~h}$ before birth and less than one week from birth. Pregnancy induced hypertension was diagnosed when an arterial blood pressure $>140$ / 90 Torr was measured repeatedly. The mode of delivery, insufficiency of the placenta, preterm labor, and a pathological cardiotocogram were additionally recorded. Insufficiency of the placenta was detected by pathological flow velocity waveforms in the umbilical artery. Pathological cardiotocogram was observed and described by deceleration, lack of acceleration, and increased baseline fetal heart rate over $170 \mathrm{bpm}$.

Neonatal characteristics of study infants included the following variables: Apgar score below 7 after 5 min, umbilical artery pH below 7.1, need for intubation and days on mechanical ventilation (including continuous positive airway pressure), intratracheal surfactant administration, RDS (based on radiographic criteria [5]), duration of oxygen dependency (days), oxygen requirements or mechanical ventilation for pulmonary reasons on day 28 (i.e., bronchopulmonary dysplasia), duration of mechanical ventilation ( $<3$ days, 3-7 days, and $>7$ days), intraventricular hemorrhage grades III and IV (according to the criteria of Papile et al. [11]), periventricular leukomalacia [9], necrotizing enterocolitis [3], retinopathy of prematurity diagnosed according to the criteria of the International Committee [10], and patent ductus arteriosus [7]. Probable sepsis was defined as the presence of characteristic clinical signs and typical laboratory data (positive blood culture, abnormal differential blood count or increased C-reactive protein). This definition did not allow us to distinguish between neonatal sepsis with or without positive blood culture. Furthermore, the neonatal mortality, i.e., death until day 28 , and the total number of hospital days until discharge were compared between both groups.

We used the SPSS statistical program (version 8.0) for statistical analyses. To compare differences between groups, $t$-tests were used for continuous variables when normally distributed; otherwise the Mann-Whitney U-test was used. Dichotomous variables were analyzed by $\chi^{2}$-test. The level of significance was set at $p<0.05$. The effect of the variables preterm labor, preterm PROM, placental insufficiency, pregnancy induced hypertension, maternal age, multiple pregnancy, and chorioamnionitis on the risk of delivering an SGA neonate were investigated by multiple logistic regression analyses. The effect of birth weight below the 10th percentile and other possible risk factors on bronchopulmonary dysplasia or death was investigated by multiple logistic regression analyses as well. The level of significance was set at $p<0.05$. Results of the multivariable analyses were expressed as odds ratios (ORs) with 95\% confidence intervals (CIs).

The present study was approved by the review board of the Institute of Quality Assurance of the Federal State of Hesse, Germany.

\section{Results}

A total of 37,393 neonates were reported by Hessian pediatric hospitals between January 1990 and December 1996 to the Hessian neonatal database and 2,341 (6.3\%) out of these were born before 32 weeks of gestation. Variables obtained within the perinatal as well as within the neonatal datasets coincided in 1,365 (58.3\%) infants out of these 2,341. Thus, a total of 1,365 infants were included in this analysis. There were no statistically significant differences in the variables gestational age, birth weight, being born SGA, RDS, bronchopulmonary dysplasia, and mortality between all 2,341 infants born below 32 weeks of gestation and the preterm infants whose perinatal and neonatal datasets could be connected (data not shown). There were also no statistically significant differences between all SGA infants below 32 weeks gestation and those with combined peri- and neonatal datasets with respect to basic neonatal and outcome variables (mean [SD] gestational age: 28.8 [1.8] vs. 28.9 [1.7] weeks; neonatal mortality: 24.2 vs. $23 \%$; RDS: 57.3 vs. $53.9 \%$; bronchopulmonary dysplasia: 21.8 vs. 28.4\%). Out of the total of 1,365 infants born before 32 weeks of gestation, $183(13.4 \%)$ neonates were SGA. The basic perinatal characteristics of SGA infants and AGA infants are given in Table 1 . The neonatal mortality was significantly higher in SGA infants.

Among all variables included in the multivariable analysis identifying risk factors for delivering an SGA neonate, only the variable placental insufficiency was associated with an increased risk for delivering an SGA neonate (OR 6.6, CI 4.2, 10.2).

The neonatal morbidity of the surviving infants is shown in Table 2. The mean duration of mechanical 
Table 1 Basic perinatal variables in small for gestational age (SGA) infants and appropriate for gestational age (AGA) infants
Table 2 Neonatal morbidity in surviving SGA infants and AGA infants

\begin{tabular}{lcrr}
\hline & SGA infants $(n=183)$ & AGA infants $(n=1,182)$ & \multicolumn{1}{c}{$p$} \\
\hline Gestational age (weeks) & $28.9(1.7)^{\mathrm{a}}$ & $28.8(2.1)^{\mathrm{a}}$ & 0.731 \\
Birth weight (g) & $788.7(178.7)^{\mathrm{a}}(380-1,230)^{\mathrm{b}}$ & $1,259.5(348.1)^{\mathrm{a}}(440-2,445)^{\mathrm{b}}<0.001$ \\
Gender (male/female); $n(\%)$ & $107 / 76(59 / 41)$ & $640 / 542(54 / 46)$ & 0.300 \\
Maternal age (years) & $28.7(5.0)^{\mathrm{a}}$ & $29.1(5.7)^{\mathrm{a}}$ & 0.889 \\
Prenatal corticosteroids; $n(\%)$ & $105(57)$ & $669(57)$ & 0.873 \\
Placental insufficiency; $n(\%)$ & $85(46)$ & $103(9)$ & $<0.001$ \\
Pregnancy induced hypertension; & $43(24)$ & $85(7)$ & $<0.001$ \\
$\quad n(\%)$ & $54(30)$ & $128(11)$ & $<0.001$ \\
Preeclampsia; $n(\%)$ & $279(24)$ & $<0.01$ \\
Preterm PROM; $n(\%)$ & $330(28)$ & $<0.001$ \\
Pathological cardiotocogram; & $110(60)$ & & $<96(59)$ \\
$\quad n(\%)$ & $84(46)$ & $928(79)$ & $<0.01$ \\
Preterm labor; $n(\%)$ & $217(20)$ & 0.001 \\
Cesarean section; $n(\%)$ & $371(93)$ & $34(3)$ & 0.162 \\
Apgar $(5$ min $<7) ; n(\%)$ & $37(23)$ & $128(11)$ & $<0.001$ \\
Umbilical artery pH <7.1; $n(\%)$ & $8(4)$ & $42(23)$ &
\end{tabular}

${ }^{a}$ Mean (SD)

${ }^{\mathrm{b}}$ Ranges

\begin{tabular}{|c|c|c|c|}
\hline & SGA $(n=141)$ & AGA $(n=1,054)$ & $p$ \\
\hline Respiratory distress syndrome; $n(\%)$ & $76(54)$ & $583(55)$ & 0.735 \\
\hline Surfactant therapy; $n(\%)$ & $56(44)$ & $359(38)$ & 0.858 \\
\hline Need for mechanical ventilation ${ }^{\mathrm{a}} ; n(\%)$ & $112(79)$ & $764(73)$ & 0.303 \\
\hline Days on the ventilator ${ }^{\mathrm{a}}$ & $16.4(14.9)^{\mathrm{b}}$ & $13(14.5)^{\mathrm{b}}$ & $<0.05$ \\
\hline Bronchopulmonary dysplasia; $n(\%)$ & $40(28)$ & $145(14)$ & $<0.001$ \\
\hline Hospitalization (days) & $93.3(60.9)^{b}$ & $64.7(32.9)^{\mathrm{b}}$ & $<0.001$ \\
\hline Periventricular leukomalacia; $n(\%)$ & $1(1)$ & $29(3)$ & 1.0 \\
\hline Intraventricular hemorrhage grades III and IV; $n(\%)$ & $4(3)$ & $54(5)$ & 1.0 \\
\hline Retinopathy of prematurity; $n(\%)$ & $26(18)$ & $134(13)$ & 0.385 \\
\hline Patent ductus arteriosus; $n(\%)$ & $24(17)$ & $140(13)$ & 0.367 \\
\hline Necrotizing enterocolitis; $n(\%)$ & $12(9)$ & $53(5)$ & 0.232 \\
\hline
\end{tabular}

${ }^{a}$ Includes days on continuous positive airway pressure

${ }^{\mathrm{b}}$ Mean (SD)
Table 3 Multivariable analysis predicting mortality for entire cohort $(n=1,365)$

\begin{tabular}{lll}
\hline Risk factor & Odds ratio & $95 \%$ CI \\
\hline Birth weight <10th percentile & 4.54 & $2.56,8.04$ \\
Gestational age & 0.56 & $0.50,0.62$ \\
Multiple pregnancy (twin) & 1.27 & $0.74,2.20$ \\
Multiple pregnancy ( $\geq 3$ ) & 1.19 & $0.41,3.41$ \\
Need for mechanical ventilation & 1.64 & $0.67,4.00$ \\
Intraventricular hemorrhage grade III & 3.36 & $1.80,6.30$ \\
$\quad$ and IV & & \\
Sex female & 0.64 & $0.42,0.98$ \\
Prenatal corticosteroid administration & 0.94 & $0.75,1.18$ \\
Pregnancy induced hypertension & 0.77 & $0.40,1.47$ \\
Placental insufficiency & 0.89 & $0.40,1.96$ \\
Preterm rupture of membrane & 0.82 & $0.51,1.33$ \\
Preterm labor & 1.03 & $0.67,1.61$ \\
Cesarean section & 0.90 & $0.52,1.56$ \\
Probable sepsis & 1.67 & $1.10,2.59$ \\
Periventricular leukomalacia & 0.74 & $0.41,1.35$ \\
Patent ductus arteriosus & 0.30 & $0.16,0.57$ \\
Surfactant therapy & 2.76 & $1.66,4.59$ \\
\hline
\end{tabular}

${ }^{a}$ Includes continuous positive airway pressure
Table 4 Multivariable analysis predicting bronchopulmonary dysplasia for survivors $(n=1,195)$

\begin{tabular}{lcl}
\hline Risk factor & Odds ratio & $95 \% \mathrm{CI}$ \\
\hline Birth weight $<10$ th percentile & 3.80 & $2.11,6.84$ \\
Increasing gestational age (weeks) & 0.67 & $0.60,0.76$ \\
Gender female & 0.63 & $0.41,0.95$ \\
Prenatal corticosteroid administration & 0.89 & $0.72,1.11$ \\
Preterm premature rupture of membrane & 1.16 & $0.74,1.82$ \\
Preterm labor & 0.93 & $0.61,1.42$ \\
Cesarean section & 0.83 & $0.48,1.44$ \\
Days on the ventilator $<3$ days & 7.79 & $0.89,68.06$ \\
Days on the ventilator 3-7 days & 15.16 & $1.96,117.16$ \\
Days on the ventilator $>7$ days & 63.88 & $8.51,479.39$ \\
Probable sepsis & 1.56 & $1.02,2.38$ \\
Patent ductus arteriosus & 1.49 & $0.92,2.43$ \\
Surfactant therapy & 1.57 & $1.01,2.43$ \\
Multiple pregnancy (twin) & 1.08 & $0.62,1.87$ \\
Multiple pregnancy $(\geq 3)$ & 0.37 & $0.10,1.43$ \\
\hline
\end{tabular}


ventilation as well as the incidence of bronchopulmonary dysplasia was significantly higher, and the mean duration of hospitalization was significantly longer in SGA infants compared with AGA infants.

By multivariable analyses, birth weight below the 10th percentile, low gestational age, need for ventilation including continuous positive airway pressure, and intraventricular hemorrhage grades 3 and 4 were identified as risk factors for an increased neonatal mortality (Table 3).

Birth weight below the 10th percentile, low gestational age, male gender, and duration of ventilation were shown to increase the risk of developing bronchopulmonary dysplasia (Table 4).

\section{Discussion}

Intrauterine growth restriction implicates an intrauterine growth pattern that deviates from the genetically determined growth percentile. Measuring intrauterine growth repeatedly by ultrasonography is the only way to prove a pathologic intrauterine growth pattern. Therefore, one has to consider that in our study as well as in other studies, neonates are defined as "SGA", although their birth weight is below the 10th percentile due to genetic reasons rather than due to a pathologic pregnancy. On the other hand, neonates with a pathologic intrauterine growth pattern not resulting in a birth weight below the 10th percentile are not defined as "SGA".

Only complete datasets from both, the perinatal and the neonatal survey have been taken in account in our final analysis. Considering this criterion, $58.3 \%$ of all datasets could be connected. We aimed to exclude the possibility that our results are biased by the high loss rate, by demonstrating that there were no statistically significant differences in main perinatal and neonatal characteristics and outcome variables between all 2,341 neonates and the 1,365 neonates, whose datasets could be connected. We further showed that there are no statistically significant differences in main perinatal and neonatal characteristics and outcome variables between all SGA neonates below 32 weeks of gestation and SGA neonates below 32 weeks of gestation whose perinatal and neonatal datasets could be connected.

The present study indicates being born SGA to result in an increased neonatal mortality in preterm infants. This finding is in accordance with several other studies $[2,4,8$, $12,15,16]$. Our analyses showed birth weight below the 10th percentile to be associated with an about five-fold increased risk of death during the neonatal period.

Few studies investigate the impact of SGA on developing bronchopulmonary dysplasia. We therefore aimed to obtain further data focusing on pulmonary neonatal morbidity in SGA infants $<32$ weeks based on data derived from a large representative population-based study. The incidence of RDS and need for surfactant administration did not differ between SGA infants and
AGA infants. Thus, we provide further evidence that being SGA does not protect against RDS. However, our data did show an increased pulmonary morbidity in SGA neonates below 32 weeks gestation. In addition to a significantly longer duration of ventilation, $28 \%$ within the SGA group compared with $14 \%$ within the AGA group developed bronchopulmonary dysplasia. By multivariable analyses, we identified the variable "being born SGA" to increase the risk of developing bronchopulmonary dysplasia 4-fold. This result is in accordance with a recently published study which shows intrauterine growth restriction to be one of the most important risk factors for prolonged oxygen requirements in infants born at less than 32 weeks gestation [6].

In conclusion, our data showed no differences between SGA infants and AGA infants in variables describing the early course of neonatal lung disease, i.e., RDS and need for surfactant therapy, whereas the incidence of bronchopulmonary dysplasia was significantly higher in the SGA group. SGA neonates below 32 weeks gestation thus represent a high-risk group regarding neonatal mortality and neonatal pulmonary morbidity.

\section{References}

1. American College of Obstetricians and Gynecologists (1998) Guidelines for perinatal care, 4th edn. American College of Obstetricians and Gynecologists, Washington, DC, p 135

2. Bardin C, Zelkowitz P, Papageorgiou A (1997) Outcome of small-for-gestational age and appropriate-for-gestational age infants born before 27 weeks of gestation. Pediatrics 100:E4

3. Bell MJ, Ternberg JL, Feigin RD et al (1978) Neonatal necrotizing enterocolitis. Therapeutic decisions based upon clinical staging. Ann Surg 187:1-7

4. Bernstein IM, Horbar JD, Badger GJ, Ohlsson A, Golan A (2000) Morbidity and mortality among very-low-birth-weight neonates with intrauterine growth restriction. The Vermont Oxford Network. Am J Obstet Gynecol 182:198-206

5. Couchard M, Polge J, Bomsel F (1974) Maladie des membranes hyalines. Diagnostic et surveillance radiologiques. Traitement, complications. Etude radioclinique de 589 cas. Ann Radiol $17: 669-683$

6. Egreteau L, Pauchard JY, Semama DS et al (2001) Chronic oxygen dependency in infants born at less than 32 weeks' gestation: incidence and risk factors. Pediatrics 108:E26

7. Gersony WM (1986) Patent ductus arteriosus in the neonate. Pediatr Clin North Am 33:545-560

8. Gortner L, Wauer RR, Stock GJ et al (1999) Neonatal outcome in small for gestational age infants: do they really better? J Perinat Med 27:484-489

9. Hill A, Melson GL, Clark HB, Volpe JJ (1982) Hemorrhagic periventricular leukomalacia: diagnosis by real time ultrasound and correlation with autopsy findings. Pediatrics 69:282-284

10. International Committee (1984) International classification of retinopathy of prematurity. Prepared by an international committee. Br J Ophthalmol 68:690-697

11. Papile LA, Burstein J, Burstein R, Koffler H (1978) Incidence and evolution of subependymal and intraventricular hemorrhage: a study of infants with birth weights less than 1,500 gm. J Pediatr 92:529-534

12. Piper JM, Xenakis EM, McFarland M, Elliott BD, Berkus MD, Langer O (1996) Do growth-retarded premature infants have different rates of perinatal morbidity and mortality than appropriately grown premature infants? Obstet Gynecol 87:169-174 
13. Procianoy RS, Garcia-Prats JA, Adams JM, Silvers A, Rudolph AJ (1980) Hyaline membrane disease and intraventricular haemorrhage in small for gestational age infants. Arch Dis Child 55:502-505

14. Simchen MJ, Beiner, ME, Strauss-Liviathan N et al (2000) Neonatal outcome in growth-restricted versus appropriately grown preterm infants. Am J Perinatol 17:187-192

15. Thompson PJ, Greenough A, Gamsu HR, Nicolaides KH (1992) Ventilatory requirements for respiratory distress syndrome in small-for-gestational-age infants. Eur $\mathrm{J}$ Pediatr 151:528-531
16. Tyson JE, Kennedy K, Broyles S, Rosenfeld CR (1995) The small for gestational age infant: accelerated or delayed pulmonary maturation? Increased or decreased survival? Pediatrics 95:534-538

17. Voigt M, Schneider KT, Jahrig K (1996) Analysis of a 1992 birth sample in Germany. I. New percentile values of the body weight of newborn infants. Geburtshilfe Frauenheilkd 56:550 558 